\title{
Variation in Inter-Animal Susceptibility to Noise Damage Is Associated with $\alpha 9$ Acetylcholine Receptor Subunit Expression Level
}

\author{
Anne E. Luebke ${ }^{1,2}$ and Paul K. Foster ${ }^{1}$ \\ ${ }^{1}$ Department of Otolaryngology and ${ }^{2}$ Neuroscience Program, University of Miami School of Medicine, Miami, \\ Florida 33136
}

Large intersubject variabilities in acoustic injury are known to occur in both humans and animals; however, the mechanisms underlying such differences are poorly understood. The olivocochlear efferent system has been hypothesized to play a significant role in protecting the cochlea from noise overexposure. In this study, we demonstrate that a newly developed test for determining average efferent system strength can predict intersubject variations in acoustic injury. In addition, the intersubject variability in cochlear expression of the $\alpha 9$ subunit of the nicotinic acetylcholine receptor was found to be proportional to an animals average efferent strength. Therefore, the inter-animal variability in the $\alpha 9$-containing acetylcholine receptor expression may be one mechanism contributing to the inter-animal variability in acoustic injury.

Key words: efferent; cochlea; hair cells; $\alpha 9$ subunit of the nicotinic acetylcholine receptor; distortion-product otoacoustic emissions; fast-adaptation; noise-induced hearing loss; noise susceptibility
A current dilemma in investigating acoustic trauma is the intersubject variability to noise-induced hearing loss. This well recognized variability in reaction to noise exposures is so pronounced that common vernacular has been developed in at least two languages to refer to ears that are less susceptible to noise damage as "steel" (German) or "tough" (English) ears, whereas more susceptible ears are termed "glass" (German) or "tender" (English) ears. It is estimated that acoustic overexposures may be responsible for at least 10 million cases of noise-induced hearing loss in the United States alone.

One protective mechanism against noise damage is the acoustic reflex, which uses the actions of the two middle ear muscles, the tensor tympani and stapedius, to physically dampen soundinduced oscillations of the eardrum and basilar membrane. In addition, the cochlea receives feedback information that originates from neurons in the superior olivocochlear brainstem region. The efferent olivocochlear projection from these brainstem neurons has been hypothesized to improve signal detection in the presence of noise (Winslow and Sachs, 1987, 1988), to contribute to protection from acoustic overexposures that would result in temporary hearing loss (Rajan and Johnstone, 1988), and, more recently, to contribute to protection from a permanent noiseinduced hearing loss (Maison and Liberman, 2000). A number of findings suggest that the variation in the robustness or strength of this efferent reflex may underlie the well recognized variation in acoustic susceptibility. These observations include previous demonstrations: (1) the reflex strength of the olivocochlear system, as

Received Dec. 6, 2001; revised March 6, 2002; accepted March 8, 2002.

This work was supported by United States Public Health Service Grant R01 DC03086 and the Chandler Chair fund of the University of Miami. We thank Amanda Lowrey and Dr. Jiao He for technical assistance. We also acknowledge the skillful assistance and support of Dr. Anna Lysakowski and the confocal microscopy center of the University of Illinois at Chicago.

Correspondence should be addressed to Dr. Anne E. Luebke, University of Miami School of Medicine, Miami, FL 33136. E-mail: aluebke@chroma.med. miami.edu.

Copyright (ㄷ) 2002 Society for Neuroscience $\quad 0270-6474 / 02 / 224241-07 \$ 15.00 / 0$ measured with either transient otoacoustic emissions or distortionproduct otoacoustic emissions (DPOAEs), varies considerably among normal-hearing humans (Veuillet et al., 1991; Collet et al., 1992); and (2) chronically de-efferented ears are more vulnerable to noise-induced permanent threshold shifts (Kujawa and Liberman, 1997; Zheng et al., 1997), and de-efferentation causes the intersubject variability in noise susceptibility to disappear (Patuzzi and Thompson, 1991). In addition, a recent report by Maison and Liberman (2000) in alert guinea pigs using DPOAE measures showed that the amount of olivocochlear reflex strength present at one single frequency was able to predict susceptibility to acoustic injury.

It has long been known that the intracochlear application of acetylcholine $(\mathrm{ACh})$ mimics the effects of electrically stimulating the olivocochlear efferent fibers (Bobbin and Konishi, 1971; Kujawa et al., 1993). Moreover, the $\alpha 9$ subunit of the nicotinic ACh receptor $\left(\alpha 9_{\mathrm{s}} \mathrm{nAChR}\right)$ was cloned and shown to be expressed by the targets of olivocochlear efferent fibers, i.e., the outer hair cells of the cochlea (Elgoyhen et al., 1994; Glowatzki et al., 1995; Morley et al., 1998; Simmons and Morley, 1998). In outer hair cells of the cochlea, the $\alpha 9$ subunit of the nicotinic acetylcholine receptor may act in concert with the $\alpha 10$ subunit, the newest member of the nicotinic ACh receptor family (Elgoyhen et al., 2001; Lustig et al., 2001); however, the $\alpha 9$ subunit is essential for the best-studied cholinergic effect on cochlea function, because $\alpha 9$ null mutant animals appear functionally de-efferented because they lack suppression of electrically evoked olivocochlear responses (Vetter et al., 1999). In hair cells, ligand-gated $\mathrm{Ca}^{2+}$ entry through the $\alpha 9 / \alpha 10$ receptor (Fuchs and Murrow, 1992) is coupled to $\mathrm{K}^{+}$efflux and intracellular hyperpolarization (Housley and Ashmore, 1991). This hyperpolarization of outer hair cells affects their electromotile responses and decreases their mechanical responses to sounds and elevates the auditory thresholds (Wiederhold and Kiang, 1970; Winslow and Sachs 1987, 1988).

This current study used a newly developed test to assess the average strength of an animal's efferent response (Luebke et al., 
2001). These experiments are designed to determine whether the cholinergic efferent system plays a role in protecting the cochlea from acoustical injury. Baseline distortion-product otoacoustic emissions were monitored, and average efferent strengths were determined. Animals were subsequently exposed to noise, and the amount of noise damage was determined by noting the difference in distortion-product otoacoustic emissions and the difference in the distortion-product otoacoustic emission thresholds. In addition, the average efferent strengths were calculated and compared with cochlear $\alpha 9_{\text {s }}$ nicotinic acetylcholine receptor expression.

\section{MATERIALS AND METHODS}

Animals. Twenty-one pigmented guinea pigs (strain 2/Ncr; Charles River Laboratories, Wilmington, MA), weighing 300-400 gm, were used as experimental subjects. All procedures were approved and monitored by the Institutional Animal Care and Use Committee of the University of Miami.

$\alpha 9 n A C h R$ antibody generation. An antibody (MU43f) against the proposed intracellular loop (between transmembranes III and IV) of the guinea pig $\alpha 9 \mathrm{nAChR}$ was generated in rabbits (Covance, Princeton, NJ) against a synthetic peptide (SKPKTARNKDL) conjugated to keyholelimpet-hemocyanin. The MU43f antibody was tested by both ELISA analysis and Western analysis and is specific for the $\alpha 9 \mathrm{nAChR}$ in both tissue and heterologous expression studies, and this peptide sequence is not contained in any other neuronal nicotinic AChR subunits $\left(\alpha_{2-10}\right.$, $\beta_{2-4}$ ) discovered to date.

Immunohistochemistry. The samples were fixed in $4 \%$ paraformaldehyde for $2 \mathrm{hr}$, and either whole mounts or $20 \mu \mathrm{m}$ sections were blocked with $10 \%$ normal goat serum in PBS. Sections were incubated in the anti- $\alpha 9$ MU43f antibody at 1:3000 in fresh blocking solution overnight at $4^{\circ} \mathrm{C}$, rinsed, and incubated in rhodamine-conjugated donkey anti-rabbit (Chemicon, Temecula) at 1:150 in $0.01 \mathrm{~m}$ PBS for $48 \mathrm{hr}$ at $4^{\circ} \mathrm{C}$. Endorgans were mounted using Vectashield on slides with spacers inserted so as not to crush the tissue. Slides were stored horizontally at $4^{\circ} \mathrm{C}$ in the dark and were imaged using a Zeiss (Oberkochen, Germany) LSM 510 confocal microscope at the University of Illinois at Chicago confocal microscope facility.

Western blot analysis. Guinea pig tissues were homogenized in $500 \mu \mathrm{l}$ of radioimmunoprecipitation analysis buffer $(1 \mathrm{M} \mathrm{NaCl}, 1 \mathrm{M}$ Tris, $0.1 \%$ NP-40, $0.05 \%$ deoxycholate, and $0.01 \%$ SDS) and incubated overnight at $4^{\circ} \mathrm{C}$ in the presence of a protease inhibitor cocktail (Boehringer Mannheim, Mannheim, Germany). Equal volumes of guinea pig cochlear proteins $(40 \mu \mathrm{l}, \sim 30 \mu \mathrm{g}$ of protein) or $30 \mu \mathrm{g}$ of other control tissue proteins (e.g., brain, skeletal muscle, or pituitary) were separated by SDS-PAGE on a $4-15 \%$ gradient gel. The gel was electroblotted to an Immobilon P membrane (Millipore, Bedford, MA), and Western blot was performed as described in the fast-blot protocol for Immobilon $\mathrm{P}$ membranes. Membranes were incubated for $60 \mathrm{~min}$ in PBS-1\% nonfat milk with primary antibody [MU43f anti- $\alpha 9$ nAChR antibody at 1:500 dilution or anti-oncomodulin monoclonal antibody at 1:10 dilution (gift of Dr. M. Henzl, University of Missouri School of Medicine, St. Louis, MO)]. The membranes were then washed and incubated in the appropriate secondary antibody (anti-rabbit for $\alpha 9 \mathrm{nAChR}$ or anti-mouse for oncomodulin) conjugated to horseradish peroxidase for $30 \mathrm{~min}$ (Jackson ImmunoResearch, West Grove, PA). In the final step, the membranes were washed, incubated in chemiluminescent substrate (Pierce, Rockford, IL), and exposed to film (Kodak LS; Eastman Kodak, Rochester, NY). Prestained molecular weight markers were also run in a parallel lane to allow size estimations of the protein bands (Novex, Wadsworth, $\mathrm{OH})$. The resulting autoradiographs were scanned, and band densities were determined using BioMax 1D image analysis software, version 1.6 (Eastman Kodak).

Oocyte membrane protein isolation. Membranes from Xenopus laevis oocytes were isolated according to the method developed by Parker et al. (1998). Briefly, oocytes were homogenized in buffer (in mM: 50 Tris, 120 $\mathrm{NaCl}, 5 \mathrm{KCl}, 1 \mathrm{MgCl}_{2}$, and $2.5 \mathrm{CaCl}_{2}, \mathrm{pH}$ 7.0) using a Brinkman Instruments (Westbury, NY) model PT 10/35 homogenizer. Homogenates were centrifuged at $4^{\circ} \mathrm{C}$ at $2000 \times g$ for $10 \mathrm{~min}$. The supernatant was removed and then centrifuged at $4^{\circ} \mathrm{C}$ at $45,0000 \times g$ for $20 \mathrm{~min}$. The supernatant was discarded, and the membrane pellet was resuspended in buffer. Approximately $125-150$ oocytes yielded $20 \mu \mathrm{g}$ of membrane protein.
DPOAE measurements. Guinea pigs were sedated with $40 \mu \mathrm{g} / \mathrm{kg}$ ketamine $\mathrm{HCl}$, and DPOAEs at $2 f_{1}-f_{2}$ were measured using software described previously (Martin et al., 1999), which included ear speakers (ER-2; Etymotic Research, Elkgrove Village, IL) to produce the $f_{1}$ and $f_{2}$ primaries and a commercially available emissions measuring system with an acoustic probe encased microphone assembly (ER-10B ${ }^{+}$; Etymotic Research) to measure the emitted responses. Both stimulus generation and response acquisition were under computer control. DPOAEs were obtained from both ears as standard distortion product grams using primary-tone levels ranging from 45 to $75 \mathrm{~dB}$ sound pressure level (SPL), in $5 \mathrm{~dB}$ steps, at geometric-mean (GM) frequencies of the $f_{1}$ and $f_{2}$ tones $\left[\mathrm{GM}=\left(f_{1} * f_{2}\right)^{1 / 2}\right]$ ranging, in 0.1-octave steps, from 1.4 to $17.8 \mathrm{kHz}$, in which $f_{2} / f_{1}=1.2$.

Average efferent determination. The magnitude of the efferent effect varies greatly across frequencies. To test efferent after-effects throughout a broad range of frequencies, the DPOAE differences were computed over a two-octave frequency range, i.e., from 2.8 to $11.3 \mathrm{kHz}$. Briefly, the baseline DPOAEs were measured in the test ear using monaural stimulation $\left(L_{1}=L_{2}=70 \mathrm{~dB}\right.$ SPL) before the onset of an efferent-based adaptive response to minimize any efferent effect (Liberman et al., 1996). A second test was then performed over the same frequency region with $L_{1}=L_{2}=70 \mathrm{~dB}$ SPL but, instead, with binaural stimulation with longer primary tones continuously presented for $1 \mathrm{sec}$ before measuring the DPOAEs to maximize the efferent-induced effects. To allow the efferent-based DPOAE measurements to reset, a $2.5 \mathrm{sec}$ interblock interval was allotted for both the baseline monaural and the binaural tests. The absolute value of each of the differences between the baseline and binaural stimulation DPOAE levels were then summed and averaged over the two-octave range by dividing this sum by the number of tested frequencies, with the result representing the average efferent strength for the test ear in units of decibels. The statistical package (StatView, version 4.5; Abacus Concepts, Berkeley, CA) was used to determine linear regression coefficients and $p$ values.

Average acoustic reflex determination. The difference between the $f_{1}$ tones during baseline monaural and binaural stimulation was computed, summed, and averaged over the same frequency range as the average efferent measure to provide a measure of the average acoustic reflex, which reflected a change in the ear-canal impedance that would result if the $1 \mathrm{sec}$ binaural primary-tone stimulation activated the middle ear muscles.

Noise exposure. One day after prenoise exposure, control DPOAEs were obtained, and guinea pigs were placed inside a sound-reverberant chamber in which they were allowed access to food and water ad libitum and exposed for $4 \mathrm{hr}$ to a $109 \mathrm{~dB}$ SPL octave band of noise, ranging from 2 to $4 \mathrm{kHz}$. After a 1 week recovery period, postexposure DPOAEs were measured as before to compare preexposure with postexposure DPOAE levels.

Average DPOAE loss and DPOAE threshold shift determinations. To quantify the loss in DPOAEs attributable to the noise overexposure, another measure was defined termed the average DPOAE loss, which represented the average difference between the pre-DPOAEs and postDPOAEs (elicited at $65 \mathrm{~dB}$ ) summed over the frequencies from 2.8 to $11.3 \mathrm{kHz}$, i.e., over the same frequency region used to determine the average efferent and average acoustic reflex measures. In addition, the change in isoresponse DPOAE contour "thresholds" were determined at a $3 \mathrm{~dB}$ SPL criterion. Average DPOAE threshold shifts were determined between the pre-DPOAE and post-DPOAE thresholds over the frequencies from 2.8 to $11.3 \mathrm{kHz}$.

\section{RESULTS}

The fast adaptive efferent test developed by Liberman et al. (1996) to test olivocochlear function in the cat was modified for application to the guinea pig using both monaural and binaural constant-tone stimulation. An example of the results obtained by this technique is presented in Figure $1 A$ for eliciting the DPOAE at a frequency of $5.6 \mathrm{kHz}$ stimulation. This plot shows that, similar to the cat, the efferent-induced reduction in DPOAEs was greatest with binaural than with monaural stimulation. The greater DPOAE adaptation with binaural stimulation is presumably attributable to more olivocochlear neurons being stimulated with binaural stimulation than with monaural sound stimulation. The binaural efferent effect on DPOAEs was stable within the 
A

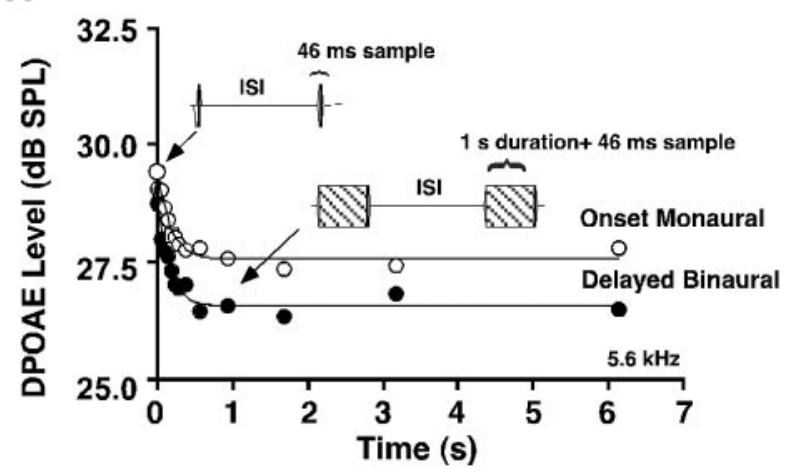

B

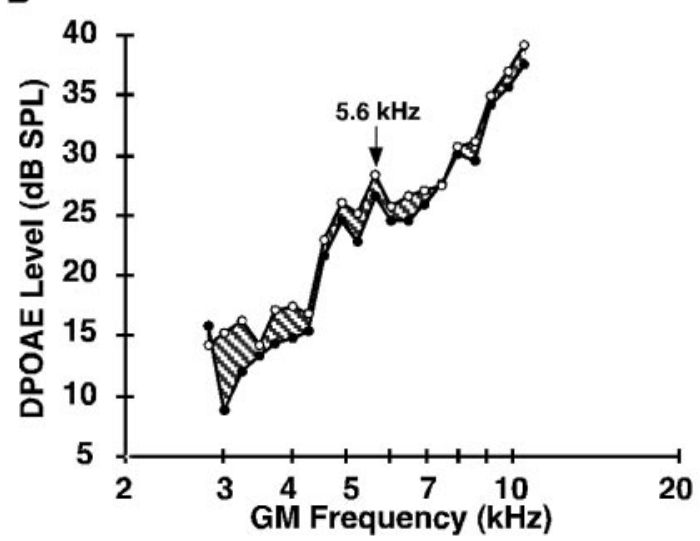

C

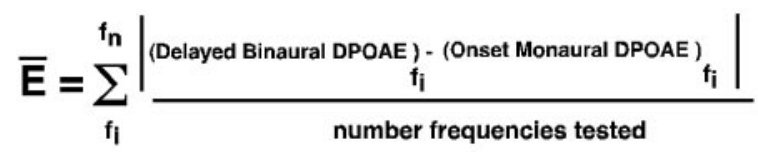

Figure 1. Binaural $1 \mathrm{sec}$ stimuli evoked the greatest efferent-induced changes in DPOAE levels. $A$, Binaural auditory stimulation at $5.6 \mathrm{kHz}$, $L_{1}=L_{2}=65 \mathrm{~dB}$ SPL, produced a larger decrement ( filled circles) than did monaural stimulation (open circles). ISI, Interstimulus interval. B, The shaded region denotes differences between baseline monaural (open circles) and binaural efferent (filled circles) stimulation in the same animal shown in $A$ for the frequency region from 2.8 to $11.3 \mathrm{kHz}\left(L_{1}=L_{2}=65\right.$ $\mathrm{dB}$ SPL) and points out the variation in efferent effects across frequencies. $C$, The formula used to determine the average efferent strength $(E)$.

same test session in which retests were performed every 5 min over a 50 min period, with variations of $<0.2 \mathrm{~dB}$. Moreover, the amount of adaptation was the same when an animal was retested $2 \mathrm{~d}$ later (data not shown). Figure $1 B$ shows the effect of binaural efferent stimulation on DPOAE levels as a function of frequency, for the same guinea pig as shown in Figure $1 \mathrm{~A}$ for a single frequency of $5.6 \mathrm{kHz}$. Figure $1 C$ shows the equation used to determine the average efferent strength for all animals tested. More details on this testing protocol can be found by Luebke et al. (2001).

The two plots of Figure $2 A$ show the difference in decibels between the baseline and the effect of binaural stimulation for one animal with a large average efferent strength [guinea pig (gp) 87L; filled squares] and one animal with a small average efferent strength (gp 89L; filled triangles). The differences between the $f_{1}$ tones with baseline and binaural stimulation were also computed over the same frequency range to provide a measure of the average acoustic reflex, which reflects an impedance change in the
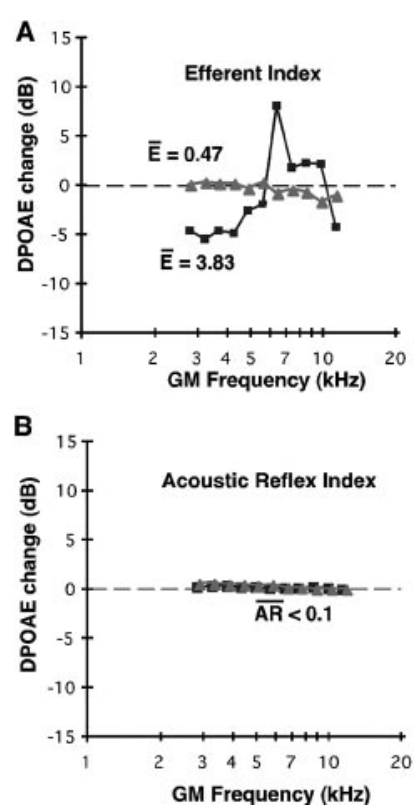

Figure 2. The strength of cochlear efferent activation was highly variable across animals. $A$, The absolute values of the difference between monaural and binaural efferent tests (see Results) were performed using equilevel tones at $70 \mathrm{~dB}$ SPL, shown here for gp 87L ( filled triangles; $E=0.47$ $\mathrm{dB}$ ) and gp $89 \mathrm{~L}$ ( filled squares; $E=3.83 \mathrm{~dB}$ ) for the frequency range from 2.8 to $11.3 \mathrm{kHz}$. $B$, The differences between the $f_{1}$ tones applied monaurally or binaurally were also computed as a measure of the average acoustic reflex $(A R)$ in the ear canal, which changed if the middle ear muscles were stimulated by the longer binaural tones, shown here for the same guinea pigs as in $A$. Ketamine sedation prevented activation of the acoustic reflex, which did not influence the average efferent measure.

ear canal if the middle ear muscles are activated by the longer tones. However, ketamine anesthesia significantly raised the acoustic reflex threshold and prevented the reflex from occurring, as shown in Figure $2 B$. Therefore, in the sedated guinea pig, the acoustic reflex did not contribute significantly to the average efferent measure.

To determine whether the average efferent strength measure can be used to predict susceptibility to noise overexposure and whether it can account for the intersubject variability in their susceptibility to noise damage, average efferent strengths were computed in a group of guinea pigs that were then subjected to the free-field octave band noise exposure, i.e., a 109 dB SPL exposure from 2 to $4 \mathrm{kHz}$ for $4 \mathrm{hr}$, delivered within a reverberant sound chamber. After a 1 week recovery period, the difference between their preexposure and postexposure DPOAEs was computed, and this difference was then summed across the tested frequency range and divided by the number of tested frequencies $(n=11)$ to yield a number reflecting each guinea pig's DPOAE loss. Figure $3 A$ shows the prenoise versus postnoise exposure DPOAEs for the animal shown previously to have a large average efferent strength in Figure $2 A$. It is evident that noise exposure did not significantly alter the DPOAEs in this subject. In contrast, exposure to the same level and duration of noise significantly reduced the DPOAEs in a second guinea pig shown previously to have a small average efferent strength (Fig. $2 B$ ). Figure $3 B$ shows DPOAE responses as a function of sound pressure level (i.e., growth response functions for both prenoise and postnoise exposure) for two frequencies ( 2 and $4 \mathrm{kHz}$ ) for the two guinea pigs shown in Figure $3 A$. As can be appreciated, the loss of gp 89 (weak efferent strength) existed for both threshold and suprath- 


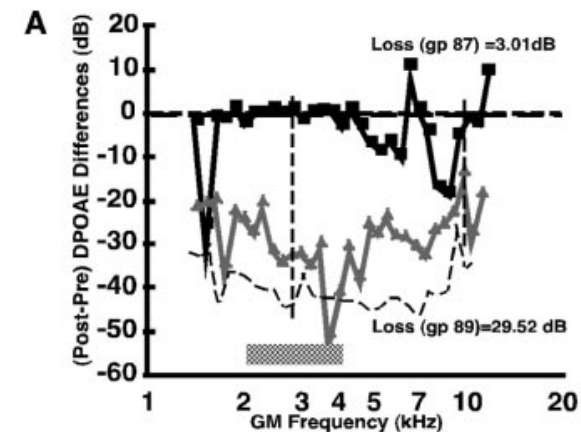

B
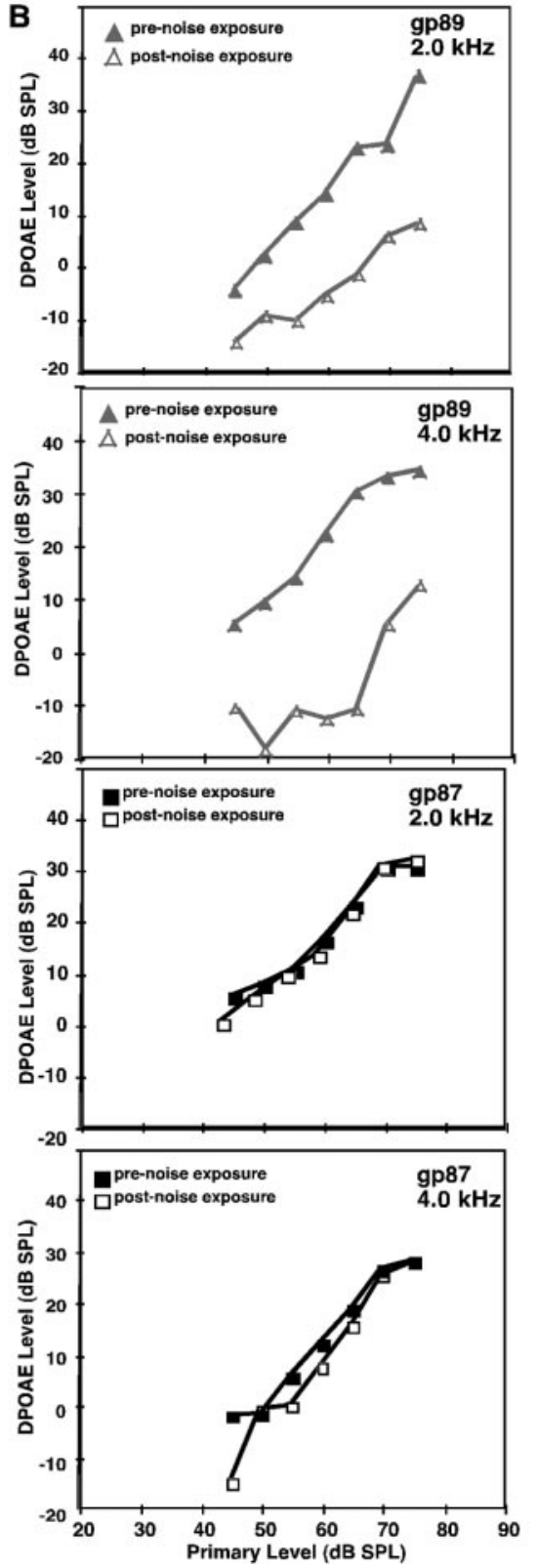

Figure 3. The amount of noise-induced hearing loss varied among animals. $A$, The average DPOAE loss was plotted as a function of DPOAE frequency to compute a value for gp 89L (filled triangles) and gp $87 \mathrm{~L}$ ( filled squares), whose average efferent strengths were shown previously. gp 89L exhibited the most DPOAE loss, with an average loss of $\sim 30 \mathrm{~dB}$, than did gp 87L, with an average loss of $3.0 \mathrm{~dB}$. $B$, DPOAE responsegrowth functions for the same animals shown in A. Filled symbols represent prenoise exposure DPAOE levels, and open symbols represent postexposure DPOAE levels.
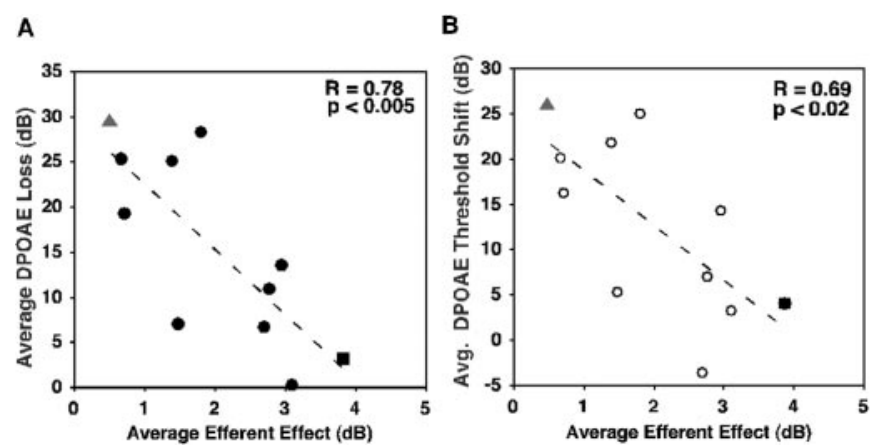

Figure 4. The strength of efferent activity predicted the amount of noise-induced hearing loss. $A$, Inverse correlation between the average efferent strength and the average DPOAE loss across guinea pig cochleas. Animals exhibiting larger efferent strengths showed less susceptibility to noise damage and had smaller DPOAE losses $(r=0.77 ; p<0.0025 ; n=$ 11 ears). Gray triangles, gp 89L; filled square, gp 87L; the remainig ears are indicated by filled circles. $B$, Inverse correlation between average DPOAE threshold shifts and average efferent strength $(r=0.60 ; p<0.02 ; n=11$ ears). Gray triangle, gp 89L; filled square, gp $87 \mathrm{~L}$; the remaining ears are indicated by open circles.

reshold sounds, whereas gp 87 (strong efferent strength) exhibited very little difference between the prenoise and postnoise exposure DPOAEs.

To quantify the loss in DPOAEs attributable to the noise overexposure, another measure termed the average DPOAE loss was used. The DPOAE loss value represents the average difference between the pre-DPOAEs and post-DPOAEs summed over the same frequency region used to determine the average efferent and acoustic reflexes. When the average DPOAE loss value was calculated for the animals shown in Figure 3, the guinea pig with a strong average efferent strength exhibited an average DPOAE loss of only $3.0 \mathrm{~dB}$ (Fig. $3 A$ ), whereas the guinea pig with a weak average efferent strength displayed an average DPOAE loss of $29.52 \mathrm{~dB}$ (Fig. 3B). For all animals, the noise-induced reduction in DPOAE levels in the form of the average DPOAE loss was analyzed at $L_{1}=L_{2}=65 \mathrm{~dB}$ SPL, which allowed the postnoise emissions to be above noise-floor levels. When the average DPOAE threshold shifts were determined for all animals tested, there was a range of DPOAE threshold shifts of $\sim 35 \mathrm{~dB}$. Again, as shown in Figure $4 B$, there was an inverse correlation between animals with weak average efferent strengths $(<1 \mathrm{~dB})$ exhibiting the great DPOAE threshold shifts $(>20 \mathrm{~dB})$ and animals with greater efferent strengths exhibiting lower threshold shifts $(<5 \mathrm{~dB})$.

The average efferent strength and the average DPOAE losses were inversely correlated for all cochleas $(n=11)$ tested, allowing a prediction of susceptibility to noise exposure $(r=0.78 ; p<$ 0.005; as shown in Fig. 4). Furthermore, the average efferent strength and the average DPOAE threshold shifts were also inversely correlated for all cochleas $(n=11)$ tested but to a lesser extent $(r=0.69 ; p<0.02)$. As shown previously in Figure 3, the animal with the greatest loss of DPOAEs after noise exposure also had the lowest average efferent value (gp 89L; filled triangles). In contrast, the animal that showed the least loss of DPOAEs after noise exposure exhibited the highest average efferent value (gp 87L; filled squares).

The presence of $\alpha 9_{\mathrm{s}} \mathrm{nAChR}$ on outer hair cells receiving efferent innervation suggests that acetylcholine signaling might be mediating the average efferent measure. Western blot analysis using an antibody against the $\alpha 9_{\mathrm{s}} \mathrm{nAChR}$ was performed to 

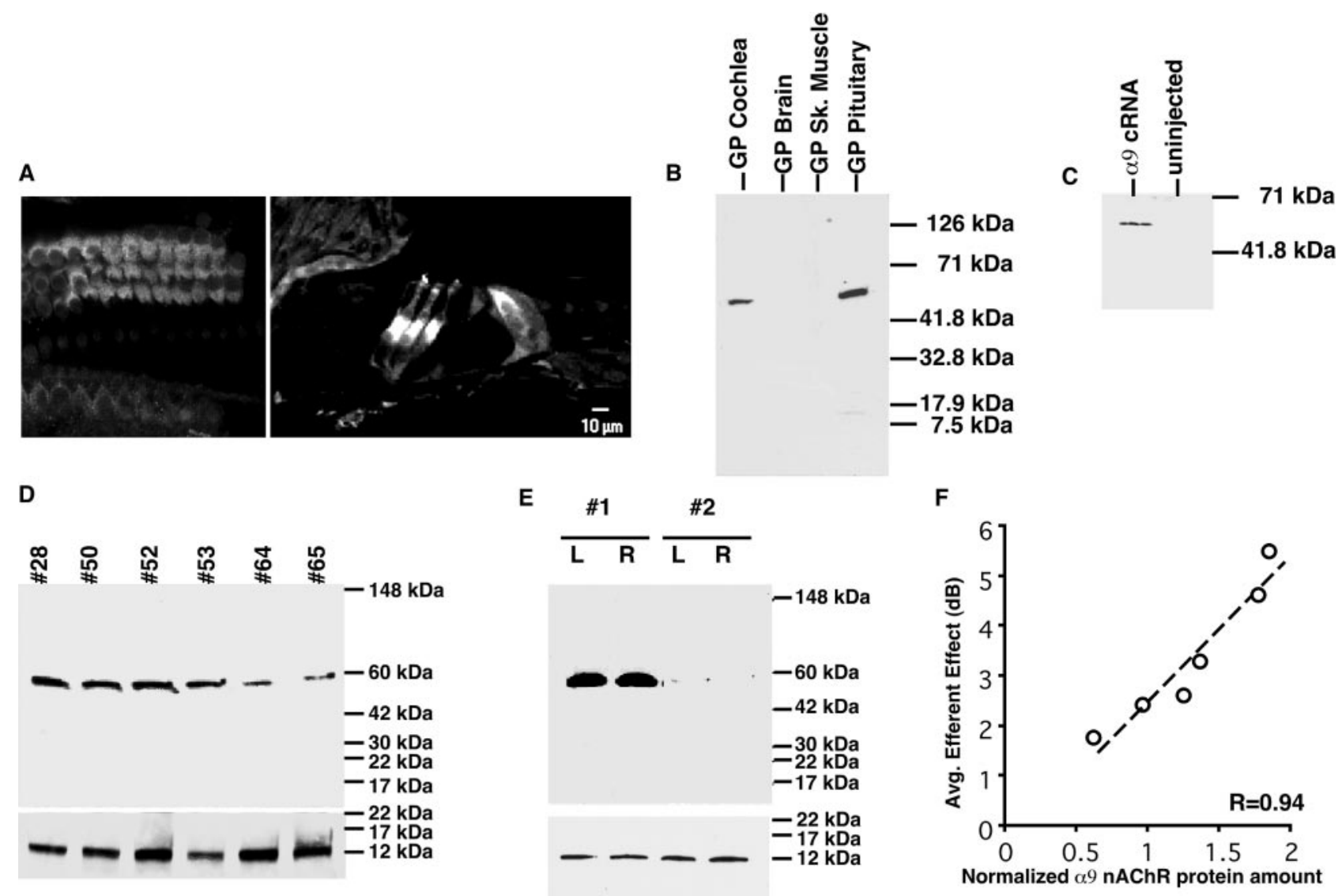

Figure 5. A given animal's average efferent strength is proportional to the amount of $\alpha 9 \mathrm{nAChR}$ present in the cochlea. $A$, Immunohistochemistry using the $\alpha 9_{\mathrm{s}} \mathrm{nAChR}$ antibody (MU43f) on cochlear sections. When the cochlea is imaged in the $\mathrm{Z}$ plane, the staining is found in the basal membrane of the outer hair cells. $\alpha 9_{\mathrm{s}} \mathrm{nAChR}$ expression in the basal portion of the three rows of outer hair cells and is present to a lesser extent in inner hair cells. $B$, $\alpha 9_{\mathrm{s}} \mathrm{nAChR}$ is expressed in the cochlea and pituitary gland but not in brain cortex or skeletal muscle tissues by Western blot analysis. $C$, Western blot using MU43f antibody detects $\alpha 9_{\mathrm{s}} \mathrm{nAChR}$ protein in membranes isolated from $\alpha 9_{\mathrm{s}}$-injected but not non-injected control $X$. laevis oocytes showed that this antibody can recognize heterogeneously expressed $\alpha 9_{\mathrm{s}} \mathrm{nAChR}$ protein. $D$, Western blot analysis showed the variability of $\alpha 9_{\mathrm{s}} \mathrm{nAChR}$ protein expression in the cochlea of six guinea pigs ( $40 \mu \mathrm{l} /$ lane, $\sim 30 \mu \mathrm{g}$ of cochlear protein). Bottom blot shows oncomodulin Western blot used to normalize for equal protein loading. $E$, Western blot analysis showing that, whereas $\alpha 9_{\mathrm{s}} \mathrm{nAChR}$ expression varies between animals (\#1, \#2), expression was equivalent between the right $(R)$ and left $(L)$ ears of the same animal $(n=4$ ears). Bottom blot shows oncomodulin Western blot showing there was no significance difference between oncomodulin protein (and hence cochlear proteins) loaded into each lane of the gel. $F, \alpha 9_{\mathrm{s}}$ nAChR protein expression correlated with the magnitude of the average efferent strength $(r=0.97 ; p<0.002 ; n=6$ ears). Blots were normalized to the amount of the calcium-binding protein oncomodulin, which is only expressed by outer hair cells (Sakaguchi et al., 1998). The amount of oncomodulin present in each cochlea sample did not vary significantly.

determine whether the average efferent strength of an intact cochlea reflected the amount of $\alpha 9_{\mathrm{s}} \mathrm{nAChR}$ present in tissue extracts of that cochlea.

The polyclonal antibody (MU43f) was specific for the $\alpha 9$ s nAChR subunit from guinea pig, and, as shown in Figure $5 A$, this antibody specifically recognized $\alpha 9_{\mathrm{s}}$ protein near the base of outer hair cells and a lesser amount of $\alpha 9_{\text {s }}$ protein immunostaining is also found in inner hair cells, consistent with in situ hybridization findings in the rat (Morley et al., 1998). We also found using immunohistochemistry that this antibody recognizes $\alpha 9_{\mathrm{s}} \mathrm{nAChR}$ in the vestibular system. In the vestibular system, type I hair cell calyces and the bases of type II hair cells contain $\alpha 9_{\text {s }}$ nAChR protein, consistent with the known location of cholinergic efferent endings (A. Luebke, P. Maroni, S. Guth, and A. Lysakowski, unpublished results).

As shown in Figure $5 B$, brain and muscle tissues did not react with this antibody and only recognized protein found in the cochlea, vestibular system, and pituitary, which are the known locations for the $\alpha 9$ nAChR subunit (Elgoyhen et al., 1994). In addition, preabsorption with the synthetic peptide eliminated the antibody reactivity (data not shown), as did incubation with the preimmune sera. The antibody did recognize $\alpha 9$ s protein from $\alpha 9$ cRNA-injected Xenopus laevis oocytes and showed no spurious bands for uninjected oocytes, as shown in Figure $5 C$. Using the MU43f antibody, the amount of $\alpha 9_{\mathrm{s}} \mathrm{nAChR}$ protein present in the cochlea was found to be highly variable among animals, as shown in Figure 5D. However, the amount of $\alpha 9_{\mathrm{s}} \mathrm{nAChR}$ protein between the two ears of the same animal was equivalent, as shown in Figure $5 E$.

The amount of $\alpha 9 \mathrm{nAChR}$ protein present in the cochlea correlated with the magnitude of the average efferent strength, as shown in Figure $5 F$, in which the magnitude of the average efferent strength is plotted against the amount of $\alpha 9 \mathrm{nAChR}$ present in the corresponding cochlea. To control for differential protein recovery or gel-loading differences, these blots were stripped and then reprobed with a monoclonal antibody generated against oncomodulin. Oncomodulin is a calcium-binding protein present only in outer hair cells of the guinea pig cochlea 
(Sakaguchi et al., 1998). Thus, normalizing the blots to the amount of oncomodulin present in each cochlea serves as a control for equal number of outer hair cells loaded into each lane. When the $\alpha 9$ s nAChR protein amount was normalized by the amount of oncomodulin present in the cochlea, the correlation between $\alpha 9 \mathrm{nAChR}$ protein and the average efferent strength measure yielded a correlation coefficient of $0.94(p<0.002 ; n=$ 6 animals), as shown in Figure $5 F$. Thus, the amount of $\alpha 9$ $\mathrm{nAChR}$ present in a given animal's cochlea correlates with the average efferent strength for that particular guinea pig.

\section{DISCUSSION}

The present study demonstrates that inter-animal variation in $\alpha 9$ nAChR cochlear protein expression correlates with the interanimal variability in the average efferent strength index. The variability in the average efferent strength index also correlates with the susceptibility to noise overexposure. Therefore, we conclude that the variation in $\alpha 9_{\mathrm{s}} \mathrm{nAChR}$ expression in adult animals correlates with differences in susceptibility to noise-induced hearing loss. The current study cannot determine whether $\alpha 9_{\mathrm{s}} \mathrm{nAChR}$ expression is mediating susceptibility or whether its expression has changed parallel to other factors. Therefore, it is not clear what causes the variability in the $\alpha 9_{\mathrm{s}} \mathrm{nAChR}$ protein expression observed in the guinea pig strain tested in the current study. Additionally, because the functional assessment of efferent reflex strength is based on olivocochlear feedback, it is not yet known whether the increased $\alpha 9 \mathrm{nAChR}$ protein levels reflect an increase in olivocochlear fibers projecting to the cochlea. In fact, when the $\alpha 9$ nAChR was knocked-out in $129 \mathrm{SvEv} / \mathrm{CBA}$ mice, those animals showed no efferent-related response when assayed using direct electrical stimulation of the efferent system (Vetter et al., 1999) and presumably would have an absent average efferent strength index. Whereas this $\alpha 9_{\mathrm{s}}$ nAChR knock-out mouse could be used to address the relationship between $\alpha 9_{\text {s }}$ nAChR expression and noise susceptibility, the mouse strain used for the knockout, and most knock-out animals generated to date, used embryonic stem cells from the $129 \mathrm{SvEv}$ mouse for the genetic recombination step. Because the $129 / \mathrm{SvEv}$ mouse strain shows exceptional resistance to acoustic injury, a straightforward test of the protective role of $\alpha 9_{\mathrm{s}} \mathrm{nAChR}$ in the knock-out mouse is confounded (Yoshida et al., 2000).

The acoustic reflex was greatly reduced in the sedated guinea pigs of the present study. Thus, the variability in activation of the acoustic reflex did not contribute significantly to the observed efferent strength variability. In accordance with these data, when Patuzzi and Thompson (1991) severed the olivocochlear bundle in guinea pigs, they noted that not only did the animals become more susceptible to acoustic injury but that the typical variability in their noise susceptibility also decreased.

In the present study, the average efferent strength was found to account for $\sim 61 \%$ of the inter-animal variability in their response to acoustic injury. This measure of efferent strength was especially effective at predicting acoustic injury for highly susceptible and weakly susceptible individuals. Perhaps the strength of each animal's acoustic reflex can account for the remaining variability in susceptibility, because the average efferent strength measurements performed in this study were determined under ketamine sedation and are not contaminated by the acoustic reflex measurement (Luebke et al., 2001). However, Maison and Liberman (2000) found that a single-frequency DPOAE measure tested on alert guinea pigs was also only $61 \%$ effective in predicting acoustic injury. Their single-frequency efferent measure was detecting a medial efferent and possibly an acoustic response, yet it also could not account for $100 \%$ of the inter-animal variability to acoustic injury. Perhaps there are other factors that we have not yet determined (i.e., strength of the lateral efferent pathway, hair cell stereocilia robustness, hair cell motility gain, etc.) that can account for the remaining inter-animal variability to acoustic injury.

The average efferent strength measure can be applied to human testing and could prove to be a useful functional test to determine both noise susceptibility and an objective diagnostic aid for brainstem-based auditory disorders. Currently, there are no objective tests for brainstem auditory impairments, yet many children may be afflicted with this problem each year, which clearly has the potential to impair their ability to integrate auditory information in noisy environments (Winslow and Sachs, 1987), thus impeding their progress in school. Because the average efferent measure specifically tests the integrity of the pathway from the brainstem to the cochlea, it can provide a reliable indicator of the functional status of that portion of the central auditory nervous system.

\section{REFERENCES}

Bobbin RP, Konishi T (1971) Acetylcholine mimics crossed olivocochlear bundle stimulation. Nat New Biol 231:222-223.

Collet L, Veuillet E, Bene J, Morgon A (1992) Effects of contralateral white noise on click-evoked emissions in normal and sensorineural ears: towards an exploration of the medial olivocochlear system. Audiology 31:1-7.

Elgoyhen AB, Johnson DS, Boulter J, Vetter DE, Heinemann S (1994) Alpha 9: an acetylcholine receptor with novel pharmacological properties expressed in rat cochlear hair cells. Cell 79:705-715.

Elgoyhen AB, Vetter DE, Katz E, Rothlin CV, Heinemann SF, Boulter J (2001) alpha10: a determinant of nicotinic cholinergic receptor function in mammalian vestibular and cochlear mechanosensory hair cells. Proc Natl Acad Sci USA 98:3501-3506.

Fuchs PA, Murrow BW (1992) Cholinergic inhibition of short (outer) hair cells of the chick's cochlea. J Neurosci 12:800-809.

Glowatzki E, Wild K, Brandle U, Fakler G, Fakler B, Zenner HP, Ruppersberg JP (1995) Cell-specific expression of the alpha 9 n-ACh receptor subunit in auditory hair cells revealed by single-cell RT-PCR. Proc R Soc Lond B Biol Sci 262:141-147.

Housley GD, Ashmore JF (1991) Direct measurement of the action of acetylcholine on isolated outer hair cells of the guinea pig cochlea. Proc R Soc Lond B Biol Sci 244:161-167.

Kujawa SG, Liberman MC (1997) Conditioning-related protection from acoustic injury: effects of chronic deefferentation and sham surgery. J Neurophysiol 78:3095-3106.

Kujawa SG, Glattke TJ, Fallon M, Bobbin RP (1993) Contralateral sound suppresses distortion product otoacoustic emissions through cholinergic mechanisms. Hear Res 68:97-106.

Liberman MC, Puria S, Guinan Jr JJ (1996) The ipsilaterally evoked olivocochlear reflex causes rapid adaptation of the 2f1-f2 distortion product otoacoustic emission. J Acoust Soc Am 99:3572-3584.

Luebke AE, Foster PK, Stagner BB (2001) A multi-frequency method for determining cochlear efferent activity. J Assoc Res Otolaryngol, in press.

Lustig LR, Peng H, Hiel H, Yamamoto T, Fuchs PA (2001) Molecular cloning and mapping of the human nicotinic acetylcholine receptor alpha10 (CHRNA10). Genomics 73:272-283.

Maison SF, Liberman MC (2000) Predicting vulnerability to acoustic injury with a noninvasive assay of olivocochlear reflex strength. J Neurosci 20:4701-4707.

Martin GK, Stagner BB, Jassir D, Telischi FF, Lonsbury-Martin BL (1999) Suppression and enhancement of distortion-product otoacoustic emissions by interference tones above $f(2)$. I. Basic findings in rabbits. Hear Res 136:105-123.

Morley BJ, Li HS, Hiel H, Drescher DG, Elgoyhen AB (1998) Identification of the subunits of the nicotinic cholinergic receptors in the rat cochlea using RT-PCR and in situ hybridization. Brain Res Mol Brain Res 53:78-87.

Parker MJ, Beck A, Luetje CW (1998) Neuronal nicotinic receptor beta 2 and beta 4 subunits confer large differences in agonist binding affinity. Mol Pharmacol 54:1132-1139.

Patuzzi RB, Thompson ML (1991) Cochlear efferent neurones and protection against acoustic trauma: protection of outer hair cell receptor current and interanimal variability. Hear Res 54:45-58.

Rajan R, Johnstone BM (1988) Binaural acoustic stimulation exercises 
protective effects at the cochlea that mimic the effects of electrical stimulation of an auditory efferent pathway. Brain Res 459:241-255.

Sakaguchi N, Henzl MT, Thalmann I, Thalmann R, Schulte BA (1998) Oncomodulin is expressed exclusively by outer hair cells in the organ of Corti. J Histochem Cytochem 46:29-40.

Simmons DD, Morley BJ (1998) Differential expression of the alpha 9 nicotinic acetylcholine receptor subunit in neonatal and adult cochlear hair cells. Brain Res Mol Brain Res 56:287-292.

Vetter DE, Liberman MC, Mann J, Barhanin J, Boulter J, Brown MC, Saffiote-Kolman J, Heinemann SF, Elgoyhen AB (1999) Role of alpha 9 nicotinic ACh receptor subunits in the development and function of cochlear efferent innervation. Neuron 23:93-103.

Veuillet E, Collet L, Duclaux R (1991) Effect of contralateral acoustic stimulation on active cochlear micromechanical properties in human subjects: dependence on stimulus variables. J Neurophysiol 65:724-735.

Wiederhold ML, Kiang NY (1970) Effects of electric stimulation of the crossed olivocochlear bundle on single auditory-nerve fibers in the cat. J Acoust Soc Am 48:950-965.

Winslow RL, Sachs MB (1987) Effect of electrical stimulation of the crossed olivocochlear bundle on auditory nerve response to tones in noise. J Neurophysiol 57:1002-1021.

Winslow RL, Sachs MB (1988) Single-tone intensity discrimination based on auditory-nerve rate responses in backgrounds of quiet, noise, and with stimulation of the crossed olivocochlear bundle. Hear Res $35: 165-189$

Yoshida N, Hequembourg SJ, Atencio CA, Rosowski JJ, Liberman MC (2000) Acoustic injury in mice: $129 / \mathrm{SvEv}$ is exceptionally resistant to noise-induced hearing loss. Hear Res 141:97-106.

Zheng XY, Henderson D, Hu BH, Ding DL, McFadden SL (1997) The influence of the cochlear efferent system on chronic acoustic trauma. Hear Res 107:147-159. 